\title{
REMARQUES SUR LA FRONTIÈRE DE MARTIN BIHARMONIQUE ET LA REPRÉSENTATION INTÉGRALE DES FONCTIONS BIHARMONIQUES
}

\author{
MOHAMED EL KADIRI AND SABAH HADDAD
}

Received 4 August 2004

Soit $(\Omega, \mathscr{H})$ un espace biharmonique fort au sens de Smyrnelis dont les espaces harmoniques associés sont des espaces de Brelot qui vérifient l'axiome de proportionnalité. On montre que s'il existe un couple $\mathscr{H}$-harmonique $>0$ sur $\Omega$, alors l'ensemble des points minimaux de la frontière de Martin biharmonique de $\Omega$ qui ne sont pas les pôles de couples biharmoniques minimaux est négiligeable dans un sens que l'on précisera. Dans le cas classique d'un domaine lipschitzien borné de $\mathbb{R}^{n}$, nous montrons que cet ensemble est vide.

\section{Introduction}

Identifiant les solutions de l'équation biharmonique classique $\Delta^{2} u=0$ dans un ouvert $\omega$ de $\mathbb{R}^{n}$ aux couples $(u, v)$ de fonctions de classe $\mathscr{C}^{2}$ dans $\omega$ telles que $\Delta u=-v$ et $\Delta v=0$, E. P. Smyrnelis a developpé dans $[13,14]$ une théorie axiomatique des fonctions biharmoniques s'appliquant plus généralement à des équations du type $L_{1} L_{2} u=0$, où $L_{1}$ et $L_{2}$ sont deux opérateurs différentiels du second ordre elliptiques ou paraboliques sur un ouvert de l'espace $\mathbb{R}^{n}$. Dans cette théorie, un espace biharmonique est la donnée d'un espace localement compact $\Omega$ et un faisceau $\mathcal{H}$ d'espaces vectoriels de couples de fonctions reélles continues sur les ouverts de $\Omega$ vérifiant certains axiomes. A un tel espace sont alors associés deux espaces harmoniques. Beaucoup de résultats des théories du potentiel, classiques ou axiomatiques, ont été étendus au cadre de la théorie des espaces biharmoniques par Smyrnelis.

Le problème de la représentation intégrale des potentiels dans un espace biharmonique fort $(\Omega, \mathscr{H})$ a été étudié par Smyrnelis dans un cadre restreint, puis par le premier auteur de ce travail, dans un cadre plus général, en utilisant la méthode des éléments extrémaux de Choquet, dans [7] où il a étudié en particulier l'ensemble des points de $\Omega$ qui ne sont pas les pôles de potentiels purs et a montré que cet ensemble est négligeable dans le sens où il est polaire relativement au deuxième espace harmonique associé à $(\Omega, \mathscr{H})$.

Le problème de la frontière de Martin a été récemment considéré par le premier auteur dans [8] où il a défini la frontière de Martin d'un espace biharmonique fort $(\Omega, \mathscr{H})$ dont les espaces harmoniques associés $\left(\Omega, \mathscr{H}_{1}\right)$ et $\left(\Omega, \mathscr{H}_{2}\right)$ sont des espaces de Brelot et vérifient 
l'axiome de proportionnalité. Cette frontière permet, comme dans le cas harmonique, de faire la représentation intégrale des couples biharmoniques positifs par des noyaux au moyen de mesures de Radon positives; le problème de Riquier relativement à cette frontière a été également étudié.

Dans le présent travail, nous étudions l'ensemble des points minimaux de la frontière de Martin $\partial^{M} \Omega$ d'un espace biharmonique fort $(\Omega, \mathscr{H})$ vérifiant les hypothèses de [8] qui ne sont pas les pôles de couples biharmoniques minimaux purs. Plus précisemment nous montrons que cet ensemble dans $\partial^{M} \Omega$ est négligeable dans un sens qui sera précisé, et nous donnons un exemple montrant qu'il peut ne pas être vide. Nous montrons aussi que si $\Omega$ est un domaine lipschitzien borné de $\mathbb{R}^{n}, n \geq 2$, muni du faisceau biharmonique associé à l'équation biharmonique classique cet ensemble est vide.

Le mot fonction signifiera toujours, sauf mention du contraire, fonction à valeurs dans $\overline{\mathbb{R}}$. Si $\left(f_{1}, g_{1}\right)$ et $\left(f_{2}, g_{2}\right)$ sont deux couples de fonctions sur un ensemble $E$, on adoptera les définitions suivantes concernant l'ordre produit usuel :

$$
\begin{aligned}
& \left(f_{1}, g_{1}\right) \geq\left(f_{2}, g_{2}\right) \Longleftrightarrow f_{1} \geq f_{2}, g_{1} \geq g_{2}, \\
& \left(f_{1}, g_{1}\right)>\left(f_{2}, g_{2}\right) \Longleftrightarrow f_{1}>f_{2}, g_{1}>g_{2},
\end{aligned}
$$

et on écrira tout simplement $(f, g) \geq 0$ (resp., $(f, g)>0)$ au lieu de $(f, g) \geq(0,0)$ (resp., $(f, g)>(0,0))$.

Les notations concernant les espaces biharmoniques seront toujours comme dans les travaux de Smyrnelis cités dans la bibliographie.

Évidemment tous les résultats de ce travail sont vrais pour un espace polyharmonique fort d'ordre quelconque; nous n'avons considéré le cas biharmonique que pour des raisons de simplicité.

\section{Résultats préliminaires}

Dans tout ce travail on se place dans un espace biharmonique fort au sens de Smyrnelis [13] $(\Omega, \mathscr{H})$ dont les espaces harmoniques $\left(\Omega, \mathscr{H}_{1}\right)$ et $\left(\Omega, \mathscr{H}_{2}\right)$ sont des espaces de Brelot et qui satisfont l'axiome de proportionnalité. Il existe alors d'après [10, proposition $22.1]$, deux noyaux de Green $G_{1}$ et $G_{2}$ relatifs aux espaces harmoniques $\left(\Omega, \mathscr{H}_{1}\right)$ et $\left(\Omega, \mathscr{H}_{2}\right)$ respectivement, c'est-à-dire,

(1) $G_{1}$ et $G_{2}$ sont deux fonctions s.c.i. sur $\Omega \times \Omega$, continues hors de la diagonale;

(2) pour tout $y \in \Omega$, la fonction $G_{i}(\cdot, y)$ est un $\mathscr{H}_{i}$-potentiel de support harmonique $\{y\}, i=1,2$.

Ces noyaux permettent de représenter les potentiels dans les espaces harmoniques $\left(\Omega, \mathscr{H}_{1}\right)$ et $\left(\Omega, \mathscr{H}_{2}\right)$ : pour tout $\mathscr{H}_{1}$-potentiel $p$ et tout $\mathscr{H}_{2}$-potentiel $q$, il existe deux mesures de Radon positives $\mu$ et $\nu$ sur $\Omega$, uniques, telles que $p=G_{1}^{\mu}$ et $q=G_{2}^{\nu}$, où, pour toute mesure de Radon $\lambda \geq 0$ sur $\Omega$, on a noté $G_{i}^{\lambda}$ la fonction définie par

$$
G_{i}^{\lambda}(x)=\int G_{i}(x, y) d \lambda(y), \quad \forall x \in \Omega .
$$

On note $U(\Omega)$ et $U_{i}(\Omega)$ (resp., $U^{+}(\Omega)$ et $U_{i}^{+}(\Omega)$ ), $i=1,2$, respectivement les cônes des couples $\mathscr{H}$-hyperharmoniques et celui des fonctions $\mathscr{H}_{i}$-hyperharmoniques (resp., $\geq 0$ ) 
dans $\Omega$. On note aussi $\mathscr{Y}^{+}(\Omega)$ et $\mathscr{Y}_{i}^{+}(\Omega), i=1,2$, respectivement les cônes des couples $\mathscr{H}_{\text {- }}$ surharmoniques et des fonctions $\mathscr{H}_{i}$-surharmoniques $\geq 0$ dans $\Omega$. Si $f$ est une fonction définie sur un ouvert $U$ de $\Omega$, on note $\hat{f}$ sa régularisée s.c.i., c'est-à-dire, la plus grande minorante s.c.i. de $f$ sur $U$.

Proposition 2.1 [14, Lemme 11.6]. Soit $v \in U_{2}^{+}(\Omega)$. Alors la fonction

$$
u_{v}=\widehat{\inf }\left\{u \in u_{1}^{+}(\Omega):(u, v) \in U^{+}(\Omega)\right\}
$$

est $\mathscr{H}_{1}$-hyperharmonique $\geq 0$ dans $\Omega$ et le couple $\left(u_{v}, v\right)$ est $\mathscr{H}$-hyperharmonique dans $\Omega$.

Définition 2.2. La fonction $u_{v}$ de la proposition précédente s'appelle la fonction hyperharmonique pure d'ordre 2 associée à $v$.

Un couple $(u, v) \in U^{+}(\Omega)$ est dit pur si $u=u_{v}$.

Remarque 2.3. S'il existe une fonction $u \in \mathscr{Y}_{1}^{+}(\Omega)$ telle que $(u, v) \in \mathscr{S}^{+}(\Omega)$ alors $u_{v} \in$ $\mathscr{Y}_{1}^{+}(\Omega)$, et c'est même un $\mathscr{H}_{1}$-potentiel.

Le théorème suivant se trouve dans [4].

THÉorème 2.4. Il existe un noyau borélien unique $\mathscr{V}$ sur $\Omega$ tel que

(i) pour toute fonction $v \in U_{2}^{+}(\Omega), \mathscr{V}(v)$ est la fonction hyperharmonique pure d'ordre 2 associée à $v$,

(ii) pour toute fonction $\varphi$ continue à support compact dans $\Omega$, la fonction $\mathscr{V}(\varphi)$ est un $\mathscr{H}_{1}$-potentiel $\mathscr{H}_{1}$-harmonique dans le complémentaire du support de $\varphi$.

Le théorème suivant, qui se trouve également dans [4], est essentiel pour la représentation intégrale des $\mathscr{H}$-potentiels et des couples $\mathscr{H}$-harmoniques $\geq 0$ dans $\Omega$.

ThÉorème 2.5. Soit $\left(s_{1}, s_{2}\right) \in \mathscr{S}^{+}(\Omega)$, alors on a $\mathscr{V}\left(s_{2}\right) \prec s_{1}$, c'est-à-dire, il existe une fonction $t \in \mathscr{S}_{1}^{+}(\Omega)$ telle que

$$
s_{1}=t+V\left(s_{2}\right)
$$

Remarque 2.6. Si $(h, k)$ est un couple biharmonique $\geq 0$ dans $\Omega$, alors $\mathscr{V}(k)$ est la partie potentielle de la fonction $h$ dans la décomposition de Riesz des fonctions $\mathscr{H}_{1}$-surharmoniques $\geq 0$ dans $\Omega$.

Soit $(p, q)$ un $\mathscr{H}$-potentiel $>0$ fini continu dans $\Omega$. La fonction $p_{0}=\mathscr{V}(q)$ est un $\mathscr{H}_{1}$ potentiel dans $\Omega$, on peut alors trouver une mesure de Radon positive $\mu_{0}$ sur $\Omega$ telle que $p_{0}=G_{1}^{\mu_{0}}$. On peut montrer que

$$
\mathscr{V}(f)=\int \frac{f(y)}{q(y)} G_{1}(\cdot, y) d \mu_{0}(y)
$$

pour toute fonction borélienne $f \geq 0$ sur $\Omega$ (voir [7]).

Exemple 2.7. Considérons l'espace biharmonique classique $\left(\mathbb{R}^{n}, \mathscr{H}\right), n \geq 1$, où le faisceau biharmonique $\mathscr{H}$ est défini pour tout ouvert $\omega$ de $\mathbb{R}^{n}$ par

$$
\mathscr{H}(\omega)=\left\{(u, v) \in\left[\mathscr{C}^{2}(\omega)\right]^{2}: \Delta u=-v, \Delta v=0\right\} .
$$


Les espaces harmoniques associés sont égaux à l'espace harmonique classique du Laplacien et vérifient donc les hypothèses de ce paragraphe. Cet espace est fort si, et seulement si, $n \geq 5$ (voir [7]). Le noyau $\mathscr{V}$ du théorème 2.4 est, dans ce cas, donné par

$$
\mathscr{V} f(x)=\frac{1}{\sigma_{n}(n-2)} \int \frac{f(y)}{\|x-y\|^{n-2}} d y
$$

pour toute fonction borélienne $f$ positive sur $\mathbb{R}^{n}$, où $\sigma_{n}$ est l'aire de la sphère unité de $\mathbb{R}^{n}$.

On sait d'après [7] que si $\Omega$ est un domaine de $\mathbb{R}^{n}, n \geq 1$, muni du faisceau biharmonique induit par $\mathscr{H}$, alors $\Omega$ est un espace harmonique fort si, et seulement si, pour tout (ou pour un) $y \in \Omega$, la fonction hyperharmonique pure d'ordre deux associée à $G_{\Omega}(\cdot, y)$ (car les espaces harmoniques associés sont symétriques) est surharmonique, où $G_{\Omega}$ est le noyau de Green de $\Omega$.

En outre, il n'est pas difficile de voir qu'un domaine borné $\Omega$ de $\mathbb{R}^{n}$ est fort et qu'il existe des couples biharmoniques purs $>0$ dans $\Omega$. Par contre, si $\Omega$ n'est pas borné, il peut ne pas exister de couples biharmoniques $>0$, comme c'est le cas si $\Omega=\mathbb{R}^{n}$ (voir [7]).

\section{Frontière de Martin biharmonique et représentation intégrale des fonctions biharmoniques}

La définition de la frontière de Martin biharmonique de $\Omega$ repose sur le théorème suivant dû à Constantinescu et Cornea (voir [9, Theorem 12.1]).

Théorème 3.1. Soit 2 une famille de fonctions continues sur $\Omega$ à valeurs dans $[-\infty,+\infty]$. Alors il existe un espace compact $\widehat{\Omega}$ tel que

(i) $\Omega$ est un ouvert dense de $\widehat{\Omega}$;

(ii) toute fonction $f$ de 2 admet un prolongement continu $f^{*}$ à $\widehat{\Omega}$;

(iii) les fonctions $f^{*}, f \in 2$, séparent les points de la frontière $\widehat{\Omega} \backslash \Omega$ de $\Omega$.

Remarque 3.2. Le théorème est vrai aussi pour une famille 2 de fonctions continues sur le complémentaire d'un même compact de $\Omega$, à condition d'imposer aux prolongements d'être continus sur le complémentaire de ce compact.

Fixons dans toute la suite un point $x_{0}$ dans $\Omega$. Pour tout $y \in \Omega$, posons

$$
\begin{aligned}
& H(x, y)= \begin{cases}\frac{G_{1}(x, y)}{G_{1}\left(x_{0}, y\right)} & \text { si } x \neq x_{0} \text { ou } y \neq x_{0}, \\
1 & \text { si } x=y=x_{0},\end{cases} \\
& L(x, y)= \begin{cases}\frac{G_{2}(x, y)}{G_{2}\left(x_{0}, y\right)} & \text { si } x \neq x_{0} \text { ou } y \neq x_{0}, \\
1 & \text { si } x=y=x_{0},\end{cases}
\end{aligned}
$$

(avec la convention $a / \infty=0$ pour tout réel $a \neq 0$ ) et considérons la famille

$$
\mathscr{2}=\{H(x, \cdot) ; x \in \Omega\} \cup\{L(x, \cdot) ; x \in \Omega\} .
$$

Les fonctions de la famille 2 sont continues dans le complémentaire de $\left\{x_{0}\right\}$. 
Définition 3.3. Le compactifié $\widehat{\Omega}$ du théorème 3.1 de $\Omega$ correspondant à la famille 2 précédente est appelé le compactifié de Martin biharmonique de $\Omega$; la frontière de $\Omega$ dans $\widehat{\Omega}$ est appelée la frontière de Martin biharmonique de $\Omega$ et notée $\partial^{M} \Omega$ (on a donc $\left.\partial^{M} \Omega=\widehat{\Omega} \backslash \Omega\right)$.

Pour tout $x \in \Omega$ on note encore $H(x, \cdot)$ et $L(x, \cdot)$ les prolongements des éléments de la famille 2 à l'espace $\widehat{\Omega}$. Il résulte alors de la propriété de Harnack dans chacun des espaces harmoniques $\left(\Omega, \mathscr{H}_{1}\right)$ et $\left(\Omega, \mathscr{H}_{2}\right)$ que, pour tout $Y \in \partial^{M} \Omega$, les fonctions $H(\cdot, Y)$ et $L(\cdot, Y)$ sont respectivement $\mathscr{H}_{1}$-harmonique $>0$ et $\mathscr{H}_{2}$-harmonique $>0$ dans $\Omega$. Pour tout $Y \in \partial^{M} \Omega$ on pose

$$
K(\cdot, Y)=\mathscr{V}(L(\cdot, Y))
$$

Remarques 3.4. (1) Dans le cas où il n'existe pas de couple $\mathscr{H}$-biharmonique $(h, k)>0$ dans $\Omega$, la frontière de Martin de l'espace harmonique $\left(\Omega, \mathscr{H}_{1}\right)$ suffirait pour représenter les couples biharmoniques $\geq 0$ puisque ces couples se réduisent, dans ce cas, aux couples de la forme $(h, 0)$ avec $h \mathscr{H}_{1}$-harmonique $\geq 0$.

(2) $\mathrm{Si} \mathscr{H}_{1}=\mathscr{H}_{2}$, la frontière de Martin biharmonique de $\Omega$ se réduit aussi à la frontière de Martin de l'espace harmonique $\left(\Omega, \mathscr{H}_{1}\right)$.

Proposition 3.5 [8, proposition 3.3]. Soit $k$ une fonction $\mathscr{H}_{2}$-harmonique $\geq 0$ dans $\Omega$. Si la fonction $\mathscr{V}(k)$ est surharmonique alors le couple $(\mathscr{V}(k), k)$ est biharmonique dans $\Omega$. En particulier, si $Y \in \partial^{M} \Omega$ est tel que $K(\cdot, Y)$ est surharmonique, alors le couple $(K(\cdot, Y)$, $L(\cdot, Y))$ est biharmonique.

Corollaire 3.6. L'ensemble $\left\{Y \in \partial^{M} \Omega ; K(\cdot, Y) \equiv+\infty\right\}$ est un borélien de $\partial^{M} \Omega$.

Démonstration. Soit $x \in \Omega$. D'après la proposition précèdente, on a

$$
\left\{Y \in \partial^{M} \Omega ; K(\cdot, Y) \equiv+\infty\right\}=\left\{Y \in \partial^{M} \Omega ; K(x, Y) \equiv+\infty\right\}
$$

D'autre part, il résulte de la définition de $K(\cdot, Y)$ et du lemme de Fatou que l'application $Y \longmapsto K(x, Y)$ est s.c.i. sur $\partial^{M} \Omega$, d'où le résultat.

Théorème 3.7 [8, théorème 3.5$]$. Soit $(h, k)$ un couple biharmonique $\geq 0$, alors il existe deux mesures de Radon $\mu$ et $\nu$ positives sur $\partial^{M} \Omega$ telles que

$$
h=\int H(\cdot, Y) d \mu(Y)+\int K(\cdot, Y) d \nu(Y), \quad k=\int L(\cdot, Y) d \nu(Y) .
$$

Corollaire 3.8. Pour qu'il existe un couple biharmonique $(h, k)>0$ dans $\Omega$ il faut et il suffit qu'il existe $Y \in \partial^{M} \Omega$ tel que $K(\cdot, Y)<+\infty$.

Dans toute la suite on suppose qu'il existe un couple biharmonique $(h, k)>0$ dans $\Omega$. Il faut noter que ce cas n'a pas toujours lieu. En effet, il suffit de prendre l'espace biharmonique classique ( $\mathbb{R}^{n}, \mathscr{H}$ ) de l'exemple 2.7. Alors, pour tout entier $n \geq 1$, il n'existe pas de couple biharmonique $(h, k)>0$ dans $\mathbb{R}^{n}$ (voir [5, exemple 1 du paragraphe 3] ).

En général on n'a pas l'unicité de la représentation intégrale dans le théorème précédent. Pour avoir l'unicité il faut imposer certaines conditions aux mesures $\mu$ et $\nu$ 
et aux fonctions $K(x, \cdot)$ et $L(x, \cdot), x \in \Omega$. Pour cela on introduit, par analogie avec le cas harmonique, les frontières minimales de $\Omega$ :

$$
\begin{aligned}
& \partial_{1}^{M} \Omega=\left\{Y \in \partial^{M} \Omega: H(\cdot, Y) \text { est minimale }\right\} \\
& \partial_{2}^{M} \Omega=\{Y \in \Delta: L(\cdot, Y) \text { est minimale }\} .
\end{aligned}
$$

On rappelle que, dans un espace harmonique, une fonction harmonique $\geq 0$ est dite minimale si toute fonction harmonique $\geq 0$ qui la minore au sens de l'ordre naturel lui est proportionnelle. De même on appelera couple biharmonique minimal tout couple biharmonique $(h, k) \geq 0$ tel que tout couple biharmonique $\geq 0$ qui le minore au sens de l'ordre naturel produit lui est proportionnel.

En considérant des bases compactes dans les cônes des fonctions $\mathscr{H}_{i}$-harmoniques positives, $i=1,2$, on montre, comme dans le cas harmonique, que les ensembles $\partial_{1}^{M} \Omega$ et $\partial_{2}^{M} \Omega$ sont des boréliens de $\partial^{M} \Omega$.

Théorème 3.9 [8, théorème 3.6]. Soit $Y \in \partial_{2}^{M} \Omega$. Si $K(\cdot, Y)<+\infty$, alors le couple $(K(\cdot, Y)$, $L(\cdot, Y))$ est minimal.

Théorème 3.10 [8, théorème 3.7]. Soit $(h, k)$ un couple biharmonique minimal, alors il existe un point $Y \in \partial^{M} \Omega$ et un réel $\alpha \geq 0$ tel que

(i) $h=\alpha H(\cdot, Y)$ si $k=0$;

(ii) $(h, k)=\alpha(K(\cdot, Y), L(\cdot, Y))$ si $k \neq 0$.

Théorème 3.11 [8, théorème 3.10]. Si les fonctions $H(x, \cdot), x \in \Omega$, séparent les points de $\partial_{1}^{M} \Omega$ et si les fonctions $L(x, \cdot), x \in \Omega$, séparent les points de $\partial_{2}^{M} \Omega$, alors pour tout couple biharmonique $(h, k) \geq 0$ dans $\Omega$, il existe deux mesures de Radon $\mu$ et $\nu$ positives uniques sur $\partial^{M} \Omega$, portées respectivement par $\partial_{1}^{M} \Omega$ et $\partial_{2}^{M} \Omega$ telles que

$$
h=\int H(\cdot, Y) d \mu(Y)+\int K(\cdot, Y) d \nu(Y), \quad k=\int L(\cdot, Y) d \nu(Y) .
$$

\section{Ensemble des pôles des couples biharmoniques minimaux purs}

On rappelle (voir [6] pour le cas classique) que si $v$ est $\mathscr{H}_{i}$-surharmonique $\geq 0$ dans $\Omega$, $i=1,2$, et si $A \subset \Delta$, on a noté [] $v[]^{A}$ la fonction égale à

$$
\inf \left\{u \in \mathcal{U}_{i}^{+}(\Omega): \exists A_{0} \text { voisinage de } A \text { dans } \widehat{\Omega} ; u \geq v \text { dans } A_{0} \cap \Omega\right\} \text {. }
$$

Il n'est pas difficile de voir que la fonction []$v[]^{A}$ est $\mathcal{H}_{i}$-harmonique dans $\Omega$.

Lemme 4.1. Pour tout $i=1,2$ et tout $A \subset \partial_{i}^{M} \Omega$, on a []$u[]^{A}=0$ ou []$u[]^{A}=u$ pour toute fonction $\mathscr{H}_{i}$-harmonique minimale $u$.

On dit que $Y \in \partial_{1}^{M} \Omega$ (resp., $Y \in \partial_{2}^{M} \Omega$ ) est un pôle d'une fonction $\mathscr{H}_{1}$ (resp., $\mathscr{H}_{2}$ )harmonique minimale $u$ (resp., $v$ ) si []$u[]^{\{Y\}}=u$ (resp., [] $v[]^{\{Y\}}=v$ ). Il faut noter que l'on a []$u[]^{A}=u$ (resp., [] $\left.v[]^{A}=v\right)$ si, et seulement si, le pôle de $u$ (resp., $v$ ) appartient à $A$. On dit que $Y \in \partial_{1}^{M} \Omega \cup \partial_{2}^{M} \Omega$ est un pôle d'un couple biharmonique minimal $(u, v)$ si $Y$ est un pôle de $v$ si $v \neq 0$, ou $Y$ est un pôle de $u$ si $v=0$. 
Lemme 4.2. Si $u=\int H(\cdot, Y) d \mu(Y)$ (resp., $v=\int L(\cdot, Y) d \mu(Y)$ ) où $\mu$ est une mesure de Radon $\geq 0$ sur $\partial^{M} \Omega$ portée par $\partial_{1}^{M} \Omega$ (resp., $\partial_{2}^{M} \Omega$ ), alors on a []$u[]^{A}=\int[] H(\cdot, Y)[]^{A} d \mu(Y)$ (resp., []$v[]^{A}=\int[] L(\cdot, Y)[]^{A} d \mu(Y)$ ).

On note $H_{f}^{1, h}$ et $H_{g}^{2, k}$ la $h$-solution et la $k$-solution du problème de Dirichlet dans les espaces harmoniques $\left(\Omega, \mathscr{H}_{1}\right)$ et $\left(\Omega, \mathscr{H}_{2}\right)$, relativement à la compactification $\hat{\Omega}$, pour les données frontières $f$ et $g$ lorsqu'elles sont respectivement $h$ - $\mathscr{H}_{1}$-résolutive et $k$ - $\mathscr{H}_{2}$ résolutive (voir par exemple [8]).

Lemme 4.3 [8, lemme 5.6]. Pour toute fonction $\mathscr{H}_{i}$-harmonique $u>0, i=1,2$, on a

$$
H_{1_{A}}^{i, u}=\frac{[] u[]^{A}}{u} .
$$

Proposition 4.4. Pour toute fonction $\mathscr{H}_{i}$-harmonique $u>0, i=1,2$, et pour tout $x \in \Omega$, la fonction

$$
A \subset \partial^{M} \Omega \longmapsto[] u[]^{A}(x)
$$

est une capacité de Choquet sur $\partial^{M} \Omega$.

Démonstration. La proposition résulte aussitôt du lemme précédent et du fait que la fonction

$$
A \subset \partial^{M} \Omega \longmapsto H_{1_{A}}^{i, u}(x)
$$

est une capacité de Choquet qui se démontre comme le résultat classique analogue dans [6, Chapter VIII, Section 6, page 111].

Définition 4.5. Soit $u$ une fonction $\mathscr{H}_{i}$-harmonique $>0$ sur $\Omega, i=1,2$. Un ensemble $A \subset$ $\partial_{i}^{M} \Omega$ est dit $u$-polaire si pour tout (ou un) $x \in \Omega$, on a []u[] ${ }^{A}(x)=0$.

Maintenant nous pouvons démontrer le résultat suivant.

Théorème 4.6. Pour toute fonction $\mathscr{H}_{2}$-harmonique $k>0$ telle que $\mathscr{V}(k)<+\infty$, l'ensemble

$$
A=\left\{Y \in \partial_{2}^{M} \Omega: K(\cdot, Y) \equiv+\infty\right\}
$$

est $k$-polaire.

Démonstration. L'ensemble $A$ est un borélien de $\partial^{M} \Omega$ d'après le corollaire de la Proposition 3.5. Soient $\mu$ et $\nu$ les mesures associées au couple $(\mathscr{V}(k), k)$ dans le théorème 3.11 (on a évidemment $\mu=0$ ). Alors, d'après le lemme 4.2 , on a

$$
[[k]]^{A}=\int[] L(\cdot, Y)[]^{A} d \nu(Y) .
$$

Or on a []$L(\cdot, Y)[]^{A}=0$ pour tout $Y \notin A$ et []$L(\cdot, Y)[]^{A}=L(\cdot, Y)$ pour tout $Y \in A$, d'où

$$
[[k]]^{A}=\int_{A} L(\cdot, Y) d \nu(Y),
$$


1468 Remarques sur la frontière de Martin biharmonique

et par suite, en vertu du théorème de Fubini,

$$
\mathscr{V}\left([[k]]^{A}\right)=\int_{A} K(\cdot, Y) d \nu(Y)
$$

Or comme $\mathscr{V}\left([[k]]^{A}\right) \leq \mathscr{V}(k) \leq h<+\infty$ et $K(\cdot, Y) \equiv+\infty$ pour tout $Y \in A$, il résulte de ce qui précède que $v(A)=0$, et donc []$k[]^{A}=0$.

Avec les mêmes notations que plus haut, nous avons le résultat suivant.

Théorème 4.7. Pour tout couple $\mathscr{H}$-harmonique pur $(h, k)>0, v_{k}(A)=0$.

Démonstration. En effet, on a

$$
\int_{A} L(\cdot, Y) d v_{k}(Y) \leq k
$$

d'où, d'après le théorème de Fubini,

$$
\int_{A} K(\cdot, Y) d v_{k}(Y) \leq \mathscr{V}(k)<+\infty
$$

Or on a $K(\cdot, Y) \equiv+\infty$ pour tout $Y \in A$, d'où $v_{k}(A)=0$.

On peut se demander si, sous l'hypothèse d'existence d'un couple pur $(h, k)>0$, l'ensemble $A$ du théorème 4.6 est vide. L'exemple suivant montre que ceci n'est pas vrai en général.

Exemple 4.8. Soit $\Omega$ l'espace ]0,1[ muni de la topologie induite par celle de $\mathbb{R}$. Pour tout ouvert $\omega$ de $\Omega$, on pose :

$$
\begin{gathered}
\mathscr{H}_{1}(\omega)=\left\{u \in \mathscr{C}^{2}(\omega) ;(x u)^{\prime \prime}=0\right\}, \\
\mathscr{H}_{2}(\omega)=\left\{u \in \mathscr{C}(\omega) ; u(x)=\frac{a}{x^{2}}+b \text { dans chaque composante connexe de } \omega\right\},
\end{gathered}
$$

où $\mathscr{C}(\omega)$ (resp., $\mathscr{C}^{2}(\omega)$ ) est l'espace des fonctions réelles continues (resp., de classe $\mathscr{C}^{2}$ ) sur $\omega$. On vérifie sans peine que les couples $\left(\Omega, \mathscr{H}_{1}\right)$ et $\left(\Omega, \mathscr{H}_{2}\right)$ sont des espaces harmoniques de Brelot qui vérifient l'axiome de proportionnalité. Si $\omega$ est un ouvert de $\Omega$, on pose :

$$
\mathscr{H}(\omega)=\left\{(u, v) \in \mathscr{C}^{2}(\omega) \times \mathscr{C}(\omega) ;(x u)^{\prime \prime}=-v, v \in \mathscr{H}_{2}(\omega)\right\}
$$

Alors $(\Omega, \mathscr{H})$ est un espace biharmonique dont les espaces harmoniques associés sont $\left(\Omega, \mathscr{H}_{1}\right)$ et $\left(\Omega, \mathscr{H}_{2}\right)$. D'autre part, il est facile de voir que le couple $(p, q)$, où $p(x)=1-x / 2$ et $q(x)=\inf \left(1, v_{0}(x)\right)$, où $v_{0}(x)=1 / x^{2}-1$ pour tout $\left.x \in\right] 0,1$ [, est un $\mathscr{H}$-potentiel, de sorte que $(\Omega, \mathscr{H})$ est un espace biharmonique fort. 
Les noyaux de Green $G_{1}$ et $G_{2}$, normalisés au point $1 / 2$, des espaces harmoniques $\left(\Omega, \mathscr{H}_{1}\right)$ et $\left(\Omega, \mathscr{H}_{2}\right)$ sont respectivement donnés par

$$
\begin{aligned}
& G_{1}(x, y)=\left\{\begin{array}{ll}
1 & \text { si } x \leq y, \\
\frac{y}{x} \frac{1-x}{1-y} & \text { si } y \leq x,
\end{array} \quad \text { si } 1 / 2 \leq y,\right. \\
& G_{1}(x, y)=\left\{\begin{array}{ll}
\frac{1}{y}-1 & \text { si } x \leq y, \\
\frac{1}{x}-1 & \text { si } y \leq x,
\end{array} \quad \text { si } y \leq 1 / 2\right. \\
& G_{2}(x, y)=\left\{\begin{array}{ll}
1 & \text { si } x \leq y, \\
\frac{y^{2}}{x^{2}} \frac{1-x^{2}}{1-y^{2}} & \text { si } y \leq x,
\end{array} \quad \text { si } 1 / 2 \leq y,\right. \\
& G_{2}(x, y)=\left\{\begin{array}{lc}
\frac{1}{3 y^{2}}-\frac{1}{3} & \text { si } x \leq y, \\
\frac{1}{3 x^{2}}-\frac{1}{3} & \text { si } y \leq x,
\end{array} \quad \text { si } y \leq 1 / 2 .\right.
\end{aligned}
$$

On en déduit que la frontière biharmonique de ]0,1[ coincide avec sa frontière usuelle, soit $\partial^{M} \Omega=\{0,1\}$. Le noyau $L$ est donné par

$$
L(x, 0)=\frac{1}{3 x^{2}}-\frac{1}{3}, \quad L(x, 1)=1 .
$$

On a $K(\cdot, 0)=\mathscr{V}(L(\cdot, 0)) \equiv+\infty$. En effet, si $K(\cdot, 0)<+\infty$, le couple $(K(\cdot, 0), L(\cdot, 0))$ serait biharmonique, d'où nécessairement

$$
K(\cdot, 0)(x)=\frac{\ln x}{x}+\frac{x}{2}+b
$$

où $b$ est une constante, mais ceci est impossible car cette fonction prend des valeurs $<0$ au voisinage de 0 . Par contre, on a

$$
K(\cdot, 1)(x)=\frac{1}{2}(1-x)
$$

pour tout $x \in] 0,1[$.

Il faut noter que le faisceau $\mathscr{H}$ ci-dessus sur $\Omega$ est induit par l'espace biharmonique $\left(\Omega^{\prime}, \mathscr{H}^{\prime}\right)$ de l'exemple 2 de la page 584 de [7], et que la frontière de Martin de $\Omega$ est identique à la frontière de $\Omega$ dans $\Omega^{\prime}$, et l'ensemble $\{0\}$ (complémentaire de l'ensemble des pôles de couples minimaux purs) est $\mathscr{H}_{2}^{\prime}$-polaire. Ceci nous conduit à poser les questions suivantes.

Question 4.9. Étant donné un domaine $\Omega$ de $\mathbb{R}^{n}$ dont la frontière de Martin est homéomorphe à sa frontière euclidienne. Est ce que tout point frontière de $\Omega$ est pôle d'un couple biharmonique minimal pur? 
Question 4.10. Si la réponse à la question précèdente est négative, est ce que l'ensemble des points de $\partial \Omega$, qui ne sont pas les pôles de couples biharmoniques minimaux purs, est polaire?

\section{Cas d'un domaine lipschitzien de $\mathbb{R}^{n}$}

Soit $\Omega$ un domaine lipschitzien borné de $\mathbb{R}^{n}$. Considérons l'opérateur différentiel $P$ défini sur $\Omega$ par

$$
P u=\Delta u+u, \quad \forall u \in \mathscr{C}^{2}(\Omega) .
$$

A cet opérateur est associé un faisceau de Brelot notés $\mathscr{H}_{P}$ (voir [2] par exemple) : les $u \in \mathscr{H}_{P}(\omega), \omega$ ouvert de $\Omega$, sont les solutions de classe $\mathscr{C}^{2}$ sur $\omega$ de l'équation $P u=0$; on sait aussi que $\mathscr{H}_{P}$ vérifie les propriétés d'unicité des potentiels de support ponctuels. On rappelle que d'après [10] l'effilement (au sens local) par rapport à $P$ coincide avec l'effilement classique et, de ce fait, les points irréguliers de $\partial \Omega$ relativement à $P$ coincident avec les points irréguliers au sens classique.

Notons $G_{P}$ et $G_{\Omega}$ les noyaux de Green de $\Omega$ relatifs aux opérateurs $P$ et $\Delta$ respectivement. $G_{P}$ et $G_{\Omega}$ sont supposés normalisés de sorte que $P G_{P}(\cdot, y)=\Delta G_{\Omega}=-\epsilon_{y}$, pour tout $y \in \Omega$, où $\epsilon_{y}$ est la mesure de Dirac au point $y$. Alors on peut trouver, d'après [3], une constante $c>0$ telle que

$$
\frac{1}{c} G_{P} \leq G_{\Omega} \leq c G_{P}
$$

Il est bien connu d'après un théorème de Hunt et Wheeden ([11, 12]), étendu par Ancona dans [2] à une large classe d'opérateurs du second ordre contenant l'opérateur $P$, que les frontières de Martin de $\Omega$ relatives aux opérateurs $P$ et $\Delta$ s'identifient à la frontière euclidienne $\partial \Omega$ de $\Omega$. Plus précisemment, on sait d'après [2], qu'à tout point $Y \in \partial \Omega$ correspondent deux fonctions $H(\cdot, Y)$ et $H_{P}(\cdot, Y)$ respectivement harmonique et $P$-harmoniques minimales, uniques, normalisées en un point $x_{0}$ fixé dans $\Omega$, bornées au voisinage de tout point $Y^{\prime} \in \partial \Omega$ distinct de $Y$ et tendent même vers 0 en $Y^{\prime}$ si $Y^{\prime}$ est régulier.

On déduit de (5.2) que l'on a

$$
\frac{1}{c^{2}} H_{P}(\cdot, Y) \leq H(\cdot, Y) \leq c^{2} H_{P}(\cdot, Y)
$$

D'autre part, on a, pour tout $Y \in \partial \Omega, \Delta H_{P}(\cdot, Y)=-H_{P}(\cdot, Y)$ de sorte que $\mathscr{V}_{\Omega} H_{p}(\cdot, Y)$ $=H_{p}(\cdot, Y)$, où $\mathscr{V}_{\Omega}$ est le noyau défini par

$$
\mathscr{V}_{\Omega} f(x)=\int_{\Omega} G_{\Omega}(x, y) f(y) d y
$$

pour toute fonction borélienne $\geq 0$ sur $\Omega$. Il résulte alors de $(5.3)$ que l'on a $\mathscr{V}_{\Omega} H(\cdot, Y)<$ $+\infty$ pour tout $Y \in \partial \Omega$, autrement dit, la fonction $\mathscr{V}_{\Omega} H(\cdot, Y)$ est biharmonique. 
Notons que la frontière de Martin biharmonique de l'espace biharmonique $\Omega$ muni du faisceau biharmonique défini par

$$
\mathscr{H}(\omega)=\left\{(u, v) \in \mathscr{C}^{2}(\omega): \Delta u=-v, \Delta v=0\right\}
$$

pour tout ouvert $\omega$ de $\Omega$, coincide avec $\partial \Omega$. Nous pouvons résumer ce qui précède dans l'énoncé suivant.

Théorème 5.1. Pour tout point $Y \in \partial \Omega$, la fonction $K_{Y}=\mathscr{V}_{\Omega} H(\cdot, Y)$ est biharmonique.

On voit donc que la réponse à la question 4.9 est positive dans le cas où le domaine $\Omega$ est lipschitzien borné.

Remarque 5.2. Il semble que théorème 5.1 s'étend à la classe des domaines uniformément de John qui contient strictement celle des domaines lipschitziens (voir [1]). Nous y reviendrons dans un travail ultérieur.

\section{Références}

[1] H. Aikawa, T. Lundh, and T. Mizutani, Martin boundary of a fractal domain, Potential Anal. 18 (2003), no. 4, 311-357.

[2] A. Ancona, Principe de Harnack à la frontière et problèmes de frontière de Martin, Potential Theory, (Proc. Colloq., Copenhagen, 1979), Lecture Notes in Math., vol. 787, Springer, Berlin, 1980, pp. 9-28.

[3] Comparaison des mesures harmoniques et des fonctions de Green pour des opérateurs elliptiques sur un domaine lipschitzien, C. R. Acad. Sci. Paris Sér. I Math. 294 (1982), no. 15, 505-508.

[4] N. Bouleau, Thèse de doctorat d'état es sciences, Université de Paris VI, 1979.

[5] G. Choquet, Lectures on Analysis. Vol. II : Representation Theory edited by J. Marsden, T. Lance, and S. Gelbart, W. A. Benjamin, New York, 1969.

[6] J. L. Doob, Classical Potential Theory and Its Probabilistic Counterpart, Grundlehren der Mathematischen Wissenschaften, vol. 262, Springer, New York, 1984.

[7] M. El Kadiri, Sur la représentation intégrale en théorie axiomatique des fonctions biharmoniques, Rev. Roumaine Math. Pures Appl. 42 (1997), no. 7-8, 579-589.

[8] - Frontière de Martin biharmonique et représentation intégrale des fonctions biharmoniques [Biharmonic Martin boundary and integral representation of biharmonic functions], Positivity 6 (2002), no. 2, 129-145.

[9] L. L. Helms, Introduction to Potential Theory, Pure and Applied Mathematics, vol. 22, WileyInterscience, New York, 1969.

[10] R. M. Hervé, Recherches axiomatiques sur la théorie des fonctions surharmoniques et du potentiel, Ann. Inst. Fourier (Grenoble) 12 (1962), 415-571.

[11] R. A. Hunt and R. L. Wheeden, On the boundary values of harmonic functions, Trans. Amer. Math. Soc. 132 (1968), 307-322.

[12] , Positive harmonic functions on Lipschitz domains, Trans. Amer. Math. Soc. 147 (1970), 507-527.

[13] E. P. Smyrnélis, Axiomatique des fonctions biharmoniques. I, Ann. Inst. Fourier (Grenoble) 25 (1975), no. 1, 35-97. 
1472 Remarques sur la frontière de Martin biharmonique

[14] Axiomatique des fonctions biharmoniques. II, Ann. Inst. Fourier (Grenoble) 26 (1976), no. 3, ix, 1-47.

Mohamed El Kadiri : Department de Mathématiques et d'Informatique, Faculté des Sciences, Université Mohammed V Agdal Rabat, B.P. 1014, Rabat, Maroc

E-mail address: elkadiri@fsr.ac.ma

Sabah Haddad : Department de Mathématiques et d'Informatique, Faculté des Sciences, Université Mohammed V Agdal Rabat, B.P. 1014, Rabat, Maroc 


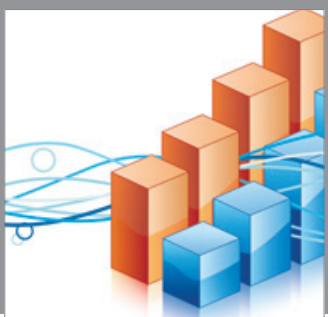

Advances in

Operations Research

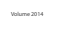

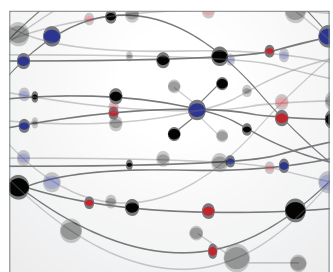

\section{The Scientific} World Journal
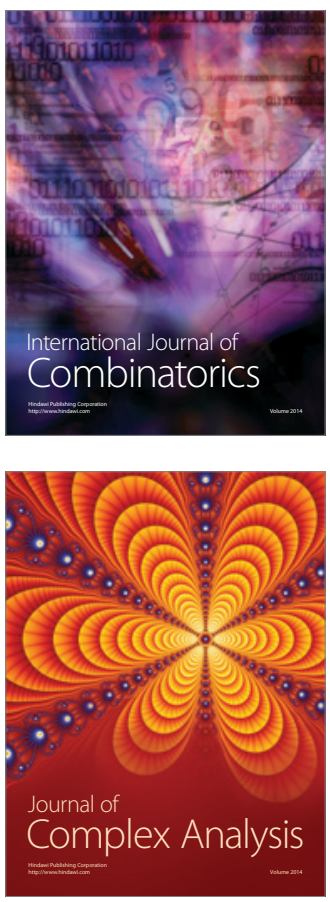

International Journal of

Mathematics and

Mathematical

Sciences
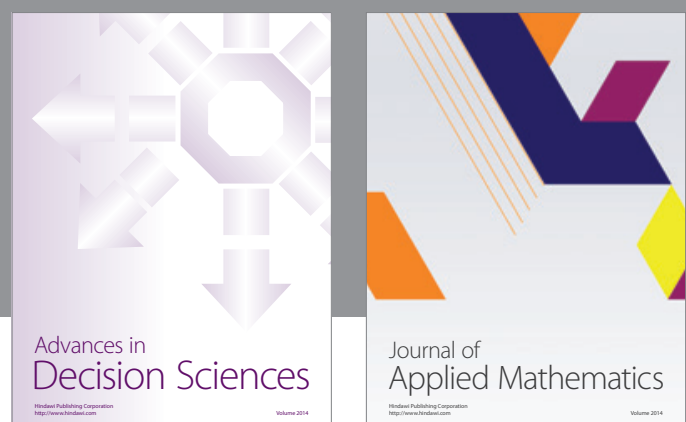

Journal of

Applied Mathematics
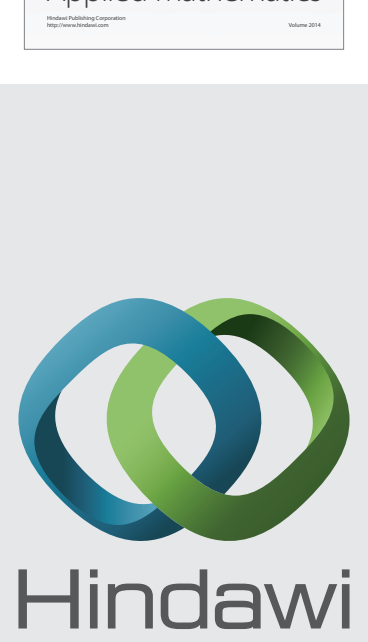

Submit your manuscripts at http://www.hindawi.com
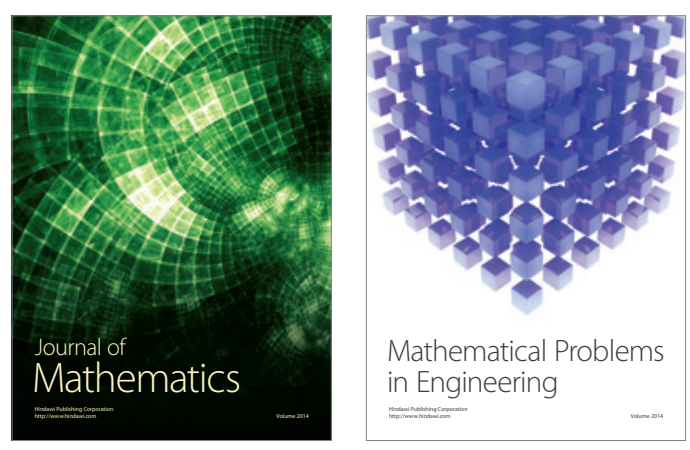

Mathematical Problems in Engineering
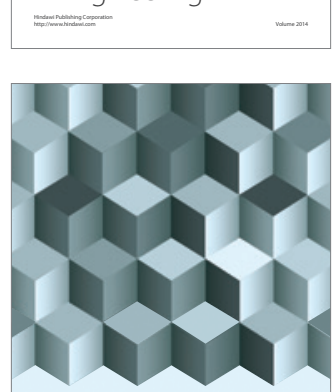

Journal of

Function Spaces
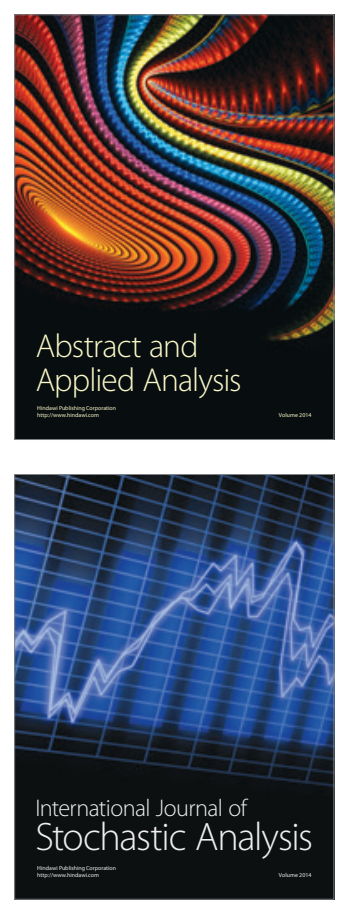

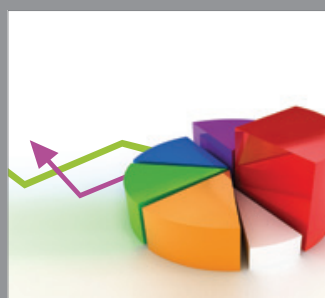

ournal of

Probability and Statistics

Promensencen
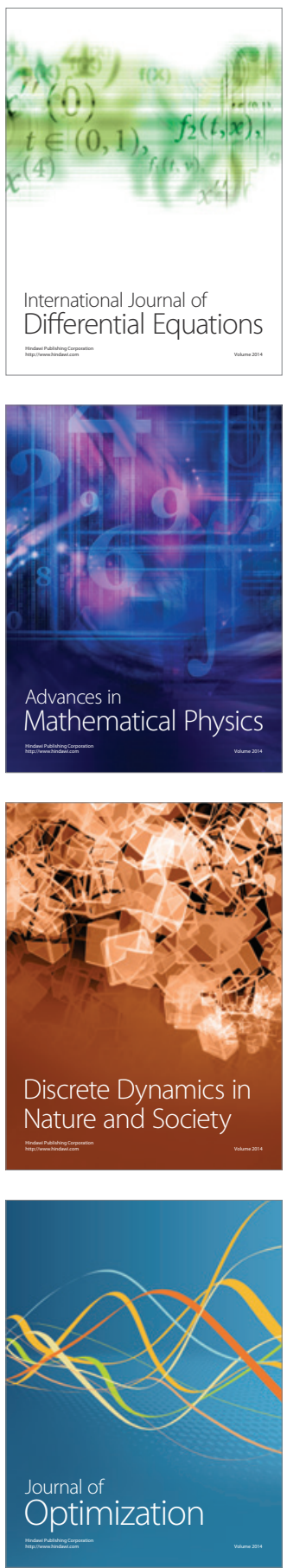